\title{
A CHARACTERIZATION OF DISCRETE BANACH LATTICES WITH ORDER CONTINUOUS NORMS
}

\author{
WITOLD WNUK \\ (Communicated by John B. Conway)
}

\begin{abstract}
We give a characterization of those $\sigma$-Dedekind complete Banach lattices for which every continuous linear operator $T: E \rightarrow c_{0}$ is a difference of two positive linear operators from $E$ into $c_{0}$.
\end{abstract}

1. Preliminary remarks. Let $E$ and $F$ be infinite dimensional Banach lattices. In general, the space $L(E, F)$ of all continuous linear operators from $E$ into $F$ is not a Riesz space (= vector lattice) with respect to the natural order, i.e., $T \geq 0$ iff $T x \geq 0$ for $x \in E_{+}$, even if $F$ is Dedekind complete. However, the subspace $L^{r}(E, F)$ of regular operators, i.e., the subspace consisting of operators which are differences of positive linear operators, is a Riesz space under the "pointwise order" provided $F$ is Dedekind complete. Moreover, $L^{r}(E, F)$ is a Banach lattice for the norm $\|T\|_{r}=\||T|\|$.

A characterization of pairs of Banach lattices $E, F$ for which $L(E, F)=L^{r}(E, F)$ (or $L(E, F) \equiv L^{r}(E, F)$, i.e., these spaces are equal and $\|T\|=\|T\|_{r}$ ) is an old problem which, in general, is still not solved. A classical result in this direction says that $L(E, F) \equiv L^{r}(E, F)$ whenever $F$ is a Dedekind complete $A M$-space with a strong unit or $E$ is an $A L$-space and there exists a positive contractive projection $P: F^{* *} \rightarrow F$. Cartwright and Lotz conjectured in [4] that if $L(E, F)=L^{r}(E, F)$, then $E$ is Riesz isomorphic to an $A L$-space or $F$ is Riesz isomorphic to an $A M$ space. They confirmed the conjecture in the case where $E^{*}$ or $F$ contains a closed sublattice Riesz isomorphic to $l^{p}$ for some $p \in[1, \infty)$, but Abramovic constructed in [1] a pair of Banach lattices $E$ and $F$ with the following properties: $E$ is not Riesz isomorphic to an $A L$-space, $F$ is not Riesz isomorphic to an $A M$-space and for any operator $T \in L(E, F)$ the modulus $|T|: E \rightarrow F$ exists.

The identity $L(E, F)=L^{r}(E, F)$ was also considered in [6] where the author, among other things, gave a characterization of a compact set $X$ provided $L(C(X), C(Y))=L^{r}(C(X), C(Y))$ for every compact set $Y$.

The space $l^{1}(A)$ is the unique Banach lattice $E$ (up to a Riesz isomorphism) having the property that $L(E, F)=L^{r}(E, F)$ for every Banach lattice $F$. Indeed, it is easy to notice that $L\left(l^{1}(A), F\right)=L^{r}\left(l^{1}(A), F\right)$ (see for example [6, Theorem 2.1]). On the other hand, if $L(E, F)=L^{r}(E, F)$ for every Banach lattice $F$ then $E$ is an $A L$-space by the result of Cartwright and Lotz. If $E$ were not discrete then by the famous Carathéodory theorem $E$ would contain a closed Riesz subspace Riesz isomorphic to $L^{1}(0,1)$. Moreover, there exists a positive projection

Received by the editors July 15, 1987 and, in revised form, August 25, 1987.

1980 Mathematics Subject Classification (1985 Revision). Primary 46B30, 47B55; Secondary $47 \mathrm{D} 15$.

Key words and phrases. Banach lattice, regular operator. 
$P: E \rightarrow L^{1}(0,1)$, and the operator $T: L^{1}(0,1) \rightarrow c_{0}$ defined by the equality

$$
T f=\left(\int_{0}^{1} f(t) \sin (n t) d t\right)_{n=1}^{\infty}
$$

is the well-known example of a continuous and nonregular operator. Therefore $T P \in L\left(E, c_{0}\right) \backslash L^{r}\left(E, c_{0}\right)$ and we have a contradiction.

For a Banach lattice $F$ with an order continuous norm it is not difficult to check that $L\left(L^{1}(0,1), F\right)=L^{r}\left(L^{1}(0,1), F\right)$ iff there exists a positive projection $P: F^{* *} \rightarrow F$. Indeed, if there did not exist a projection then $F$ could not be a band in $F^{* *}$. Thus $F$ contains a closed Riesz subspace Riesz isomorphic to $c_{0}$. Since the norm on $F$ is order continuous there exists a positive projection $P: F \rightarrow c_{0}$ (see $[8])$, and so the operator $T$ defined by $(*)$ is not regular as a map from $L^{1}(0,1)$ into $F$.

2. Main result. The purpose of this paper is to characterize those $\sigma$-Dedekind complete Banach lattices $E$ for which $L\left(E, c_{0}\right)=L^{r}\left(E, c_{0}\right)$. The lemma mentioned below is known (see for example [6]) but for the sake of completeness and reader's convenience we will present a proof of the lemma using a simpler and different method than in [6].

LEMMA. If $K$ is an infinite compact Hausdorff space then

$$
L\left(C(K), c_{0}\right) \neq L^{r}\left(C(K), c_{0}\right) .
$$

ProOF. Choose a weak* null sequence $\left(f_{n}\right)$ of norm-one functionals in the dual space of $C(K)$ (such a sequence exists by the Josefson-Niessenzwieg theorem) and define an operator $T: C(K) \rightarrow c_{0}$ by $T x=\left(f_{n}(x)\right)_{n=1}^{\infty}$. Map $T$ is continuous and noncompact. On the other hand, every regular operator from $C(K)$ into $c_{0}$ maps the unit ball, which is an order interval, into some order interval in $c_{0}$. But order intervals in $c_{0}$ are compact, and so every regular operator from $C(K)$ into $c_{0}$ must be compact. Therefore $T \in L\left(C(K), c_{0}\right) \backslash L^{r}\left(C(K), c_{0}\right)$.

COROLLARY. $L\left(l^{\infty}, c_{0}\right) \neq L^{r}\left(l^{\infty}, c_{0}\right)$.

Using the corollary we will prove the following characterization of discrete Banach lattices with order continuous norms.

THEOREM. Let $(E,\|\cdot\|)$ be a $\sigma$-Dedekind complete Banach lattice. The following statements are equivalent:

(a) $L\left(E, c_{0}\right) \equiv L^{r}\left(E, c_{0}\right)$.

(b) $L\left(E, c_{0}\right)=L^{r}\left(E, c_{0}\right)$.

(c) $E$ is discrete and the norm $\|\cdot\|$ is order continuous.

Proof. (a) $\Rightarrow$ (b) obvious; (b) $\Rightarrow$ (c). If the norm $\|\cdot\|$ is not order continuous then $E$ contains a closed Riesz subspace Riesz isomorphic to $l^{\infty}$. By injectivity of $l^{\infty}$ there exists a positive projection $P: E \rightarrow l^{\infty}$. Using the corollary choose a nonregular operator $S: l^{\infty} \rightarrow c_{0}$. There are no difficulties in verifying that the operator $S P$ is also nonregular. Therefore $\|\cdot\|$ is order continuous.

Suppose $E$ is nondiscrete. Denote by $E_{a}$ the band generated by discrete elements in $E$. Let $B(e)$ be the band in $E$ generated by a strictly positive element $e \in E_{a}^{d}$ $\left(E_{a}^{d}\right.$ means the orthogonal completion of $\left.E_{a}\right)$. The Banach lattice $(B(e),\|\cdot\|)$ has 
an order continuous norm and a weak unit, and so $B(e)$ is Riesz isomorphic to an ideal of some space $L^{1}(S, \Sigma, \mu)=L^{1}(\mu)$, where $\mu$ is nonatomic and probabilistic (see [7, Theorem 1.b.14]). Moreover, we can assume $L^{\infty}(\mu)$ is contained in the range of $B(e)$ and the characteristic function of the set $S$ is the range of $e$. We will identify $B(e)$ with its range in further considerations. Choose a separable sub- $\sigma$ algebra $\Sigma_{0}$ of $\Sigma$ such that $\mu_{0}=\mu \mid \Sigma_{0}$ (the restriction of $\mu$ to $\Sigma_{0}$ ) is nonatomic. The space $L^{1}\left(S, \Sigma_{0}, \mu_{0}\right)=L^{1}\left(\mu_{0}\right)$ is a closed Riesz subspace of $L^{1}(\mu)$. Therefore, there exists a positive projection $P: L^{1}(\mu) \rightarrow L^{1}\left(\mu_{0}\right)$. Using the famous Carathéodory theorem we can identify $L^{1}\left(\mu_{0}\right)$ with $L^{1}(0,1)$.

If $P_{0}$ denotes the band projection from $E$ onto $B(e)$ then the composition $P P_{0}$ maps continuously $E$ into $L^{1}(0,1)$ because $P P_{0}$ is a positive operator. Define the operator $T: E \rightarrow c_{0}$ by the equality $T x=\left(\int_{0}^{1} P P_{0} x(t) \sin (n t) d t\right)_{n=1}^{\infty}$. If $T$ were regular then the set $T([0, e])$ would be order bounded and therefore conditionally compact. But $T([0, e])$ contains the subset $\left\{\left(\int_{A} \sin (n t) d t\right)_{n=1}^{\infty}: A\right.$ is a Lebesgue measurable subset of $(0,1)\}$ which is not conditionally compact in $c_{0}$. Thus $T$ is continuous and nonregular and we have a contradiction. Therefore $E$ is a discrete Riesz space.

(c) $\Rightarrow$ (a). Let $\left(e_{a}\right)_{a \in A}$ be a complete disjoint system in $E$ consisting of discrete elements. For every $x \in E$ there exists the unique net $\left(t_{a}\right)$ of real numbers such that $x=\sum_{a} t_{a} e_{a}$. Let $T: E \rightarrow c_{0}$ be a linear continuous operator. Putting $S x=\sum_{a} t_{a}\left|T e_{a}\right|$ we have $S=|T|$ (operator $S$ is well defined because $c_{0}$ is an $A M$-space so the convergence of $\sum_{a} t_{a} T\left(e_{a}\right)$ implies the convergence of the series $\sum_{a}\left|t_{a} T\left(e_{a}\right)\right|$ and this convergence is unconditional). Thus $L\left(E, c_{0}\right)=L^{r}\left(E, c_{0}\right)$.

On the other hand $|T| x=\sup \{|T y|:|y| \leq x\}$ for $x \in E_{+}$. Since $c_{0}$ is super Dedekind complete there exists a sequence $\left(y_{n}\right) \subset E$ with two properties: $\left|y_{n}\right| \leq x$ and $|T| x=\sup _{n}\left|T y_{n}\right|$. Using this fact we have for $x \geq 0$,

$$
\begin{aligned}
\||T| x\| & =\left\|\sup _{k}\left(\left|T y_{1}\right| \vee \cdots \vee\left|T y_{k}\right|\right)\right\|=\lim _{k \rightarrow \infty}\left\|\left|T y_{1}\right| \vee \cdots \vee\left|T y_{k}\right|\right\| \\
& =\lim _{k \rightarrow \infty} \max _{1 \leq i \leq k}\left\|T y_{i}\right\| \leq\|T\|\|x\| .
\end{aligned}
$$

Thus $\|T\|_{r}=\|T\|$ because $\|T\|_{r} \geq\|T\|$ always holds.

REMARKS. 1. It is clear that every compact operator $T$ from an arbitrary Banach lattice into $c_{0}$ is regular (indeed, $T$ maps order intervals into relatively compact sets in $c_{0}$ which are order bounded).

2. A similar proof of implication (c) $\Rightarrow$ (a) is presented in [6] (see Theorem 2.2).

3 . The theorem also gives the following characterization of discrete $\sigma$-Dedekind complete Banach lattices with order continuous norms:

(d) The mapping $f \rightarrow|f|$ from $E^{*}$ into $E^{*}$ is $\sigma\left(E^{*}, E\right)$ sequentially continuous.

Indeed, it is not difficult to notice that statement (b) of the theorem is equivalent to (d) (but the mapping $f \rightarrow|f|$ is not $\sigma\left(E^{*}, E\right)$ continuous at zero if $\operatorname{dim} E=\infty-$ see [2] the proofs of Theorems 6.8 and 6.9).

\section{REFERENCES}

1. Ju. A. Abramovix, The space of operators that act between Banach lattices, Zap. Naurn. Sem. Leningrad. Otdel. Mat. Inst. Steklov 73 (1977), 188-192. (Russian)

2. C. Aliprantis and O. Burkinshaw, Locally solid Riesz spaces, Academic Press, New York and London, 1978. 
3. __ Positive operators, Academic Press, New York and London, 1985.

4. D. I. Cartwright and H. P. Lotz, Some characterizations of $A M$ - and $A L$-spaces, Math. Z. 142 (1975), 97-103.

5. D. H. Fremlin, A characterization of $L$-spaces, Indag. Math. 36 (1974), 270-275.

6. Hong-Yun Xiong, On whether or not $L(E, F)=L^{r}(E, F)$ for some classical Banach lattices $E$ and $F$, Indag. Math. 46 (1984), 267-282.

7. J. Lindenstrauss and L. Tzafriri, Classical Banach spaces. II, Function spaces, Springer-Verlag, Berlin and New York, 1979.

8. P. Meyer-Nieberg, Charakterisierung einiger topologischer und ordnung theoretischer Eigenschaften von Banachverbanden mit Hilfe disjunkter Folgen, Arch. Math. 24 (1973), 640-647.

Mathematical institute, Polish ACademy of Sciences, Poznań Branch, Mielżý́SKiEgo 27/29, 61-725 POZNAŃ, POLAND 